\title{
Protection requirements capture for superconducting cables in TeDP aircraft using a thermal-electrical cable model
}

\author{
S. Nolan, C. E. Jones, P. Norman, S. Galloway, G. Burt \\ University of Strathclyde, UK.
}

\begin{abstract}
Turbo-electric distributed propulsion (TeDP) for aircraft allows for the complete redesign of the airframe so that greater overall fuel burn and emissions benefits can be achieved. Whilst conventional electrical power systems may be used for smaller aircraft, large aircraft ( $\sim 300 \mathrm{pax})$ are likely to require the use of superconducting electrical power systems to enable the required whole system power density and efficiency levels to be achieved. The TeDP concept requires an effective electrical fault management and protection system. However, the fault response of a superconducting TeDP power system and its components has not been well studied to date, limiting the effective capture of associated protection requirements. For example, with superconducting systems it is possible that a hotspot is formed on one of the components, such as a cable. This can result in one subsection, rather than all, of a cable quenching. The quench transition to normal conduction leads to a temperature rise which is not uniformly distributed along the cable length. Due to the high current density and low cable mass of a TeDP system, this damaging failure mode can occur over a short timescale. To improve the understanding of the formation of this failure mode and its impact on a TeDP distribution cable, this paper presents a transient thermal-electrical model based on numerical methods. Using this approach, the model is then used to provide new information supporting the capture of speed and sensitivity requirements for TeDP protection systems.
\end{abstract}

\section{Introduction}

To meet the growing demand for air transport for both passengers and cargo, it is estimated that by 2030,32,600 new aircraft will be required [1]. Alongside meeting this growing demand, the aerospace industry must address environmental targets to reduce gas and noise emissions attributable to aircraft $[2,3]$. To achieve these goals, disruptive technological change is required in a number of fundamental areas central to the design of the aircraft. One such area is the propulsion drive train, in which TeDP can provide substantial advantages [2]. In such a system, electrical power is provided by generators driven by gas engines. This electrical power is then distributed to a number of electrical propulsor motors which provide the thrust for the aircraft. No thrust is provided by the gas engines. The use of the gas engines and propulsor motors in this way provides greater design freedom, allowing air-framers to harness aerodynamically advantageous effects such as Boundary Layer Ingestion (BLI) [4], improving overall aircraft efficiency.

For aircraft applications with lower power levels (e.g. 1-10 MW for some hybrid electric concept aircraft) it is considered probable that conventional electrical power distribution and associated technologies can be used to achieve efficiency gains [5]. However, it is proposed that for large aircraft (300 pax) which require upwards of $20 \mathrm{MW}$ electrical power at takeoff, the power density that can be achieved with conventional electrical machines is prohibitive and that superconducting electrical machines must be implemented [4]. To avoid inefficiencies due to heat sinks within a system, it is proposed that the full TeDP electrical power system be superconducting [6]. Superconducting materials can afford exceptional power densities, presenting the opportunity to reduce the size of the electrical machines by a factor of four [7]. It is critical to the design of the electrical power system that the failure modes and electrical protection requirements of these superconducting systems is understood, to ensure that an appropriate approach to electrical protection and redundancy is taken.

To operate within the superconducting state, high temperature superconducting (HTS) systems must be kept at cryogenic temperatures and must be operated below a critical current and magnetic field value. These three variables define the critical surface, the limits of the superconductor's operating conditions. Outside this region the material undergoes a fundamental phase change (quench) and becomes electrically resistive. Whilst quench provides the potential opportunity to dampen fault current through the sudden and large resistance increase of the material, large amounts of heat can be generated through this process due to Ohmic losses. If the quench is not managed through appropriate means, either through passive protection methods such as parallel shunts or active dump loads and heating mechanisms, there is potential for severe damage to the component [8].

One issue surrounding the quench behavior of superconducting components is the prospect of a hotspot occurring. A hotspot is the result of a non-uniform quench, when one part of the component ceases to be superconducting before the remainder of it. This causes localized heating and damage to the component [9]. The small size of the hot spot relative to the full size of the component means that a large current can still flow in the device. This in turn means the hotspot can be forced to dissipate large amounts of heat for a significant period of time before the normal zone spreads to the surrounding superconducting material, which will in turn increase the impedance of the device. Due to the relatively high heat capacity of high temperature superconducting components compared to their low temperature counterparts, the velocity at which the normal zone spreads throughout the superconducting component can be prohibitively slow. This can be as low as centimeters per second [10]. Due to this potential for damage, a protection system for superconducting systems must be able to detect quench quickly and under a variety of conditions. 
The small size and low propagation velocity can make hotspots very difficult to detect using conventional quench protection techniques which are reliant on detecting voltage drops across components [11]. Hotspots are most likely to occur due to overcurrent conditions that bring the superconductor to current levels near and above the boundaries of the superconducting state where current is not high enough to fully quench all parts of the material [12]. This means that it represents a failure mode that could occur at currents that components may be expected to ride through. Hence when designing the system, it may be necessary to over-rate components sufficiently to reduce the impact of this failure mode on the TeDP power network. However, increasing the amount of superconducting material used in the component will invariably impact its final cost.

This paper will use a numerical model to assess the impact of hotspots on a TeDP distribution cable. This is achieved by thermal and electrical discretization of the cable. Using this model, the paper explores the formation of hotspots on two types of superconducting cable designs: fault current tolerant (FCT) and fault current limiting (FCL), and explains some of the potential failure modes of these cables and their impact to a wider TeDP system.

\section{Development of Appropriate Cable Models}

\section{Modelling of Superconducting Cables}

An electrical-thermal model of the superconducting cable has been developed and adapted for both fault current limiting (FCL) and fault current tolerant (FCT) cable designs. Results from this model form the basis for the discussion of FCL and FCT cables later in this paper. The thermal model has been created by discretizing the superconducting tapes in the longitudinal direction. The thermal discretization is carried out in accordance with the application of the backward time, centered space method (BTCS). This allows for the modelling of heat conduction throughout the component while also providing the basis for the interleaving of different critical currents along the length of the cable. This implicit method allows for finding the solution to the equation for heat conduction within a solid. It is assumed that the cable is sufficiently insulated such that effects of the coolant can be ignored over the timescales considered in this paper, which are fractions of a second. This is expressed in Equation 1 , where $k$ is thermal conductivity $\left.(\mathrm{W} / \mathrm{K} . \mathrm{m}), C_{p}(\mathrm{~J} / \mathrm{kg})\right)$ is specific heat, $\rho\left(\mathrm{kg} / \mathrm{m}^{\wedge} 3\right)$ is the density of the material, $T(\mathrm{~K})$ is temperature, $Q$ is the heat flux generated within the body and $t(\mathrm{~s})$ is time,

$$
\frac{\partial T}{\partial t}=\frac{k}{C_{p} \rho} \frac{d^{2} T}{d x^{2}}+\frac{Q}{C_{p} \rho}
$$

Applying the central difference implicit method to Equation 1 produces the following Equation 2 where $T_{i}^{t}$ is the temperature of node $i$ at time $t$ and $Q_{i}^{t}$ is the heat flux generated at node $i$ at time $t$. This discretizes the component into smaller nodes that represent points along the cable's length:

$$
\frac{T_{i}^{t+1}-T_{i}^{t}}{\Delta t}=\frac{k\left(T_{i-1}^{t+1}-2 T_{i}^{t+1}+T_{i+1}^{t+1}\right)}{C_{p} \rho \Delta x^{2}}+\frac{Q_{i}^{t}}{C_{p} \rho}
$$

which can be rearranged into the following form:

$$
(1+2 F) T_{i}^{t+1}-F T_{i-1}^{t+1}-F T_{i+1}^{t+1}=T_{i}^{t}+\frac{\Delta t Q_{i}^{t}}{C_{p} \rho}
$$

where $\mathrm{F}$ is defined as:

$$
F=\frac{\Delta t k}{C_{p} \Delta x^{2} \rho}
$$

Once in this form it is possible to produce a sparse matrix form as shown in Equation 5 where $K$ accommodates the boundary conditions of the problem: 
In sparse matrix form the Equation can be solved at each time step using the efficient Matlab backslash operator. Heat generated is calculated according to $I^{2} R$ losses, taking into account current and resistance of the superconducting conduction layer and the conventional stabilizing and former materials that are inherent in the overall cable design.

Each node in this one dimensional model characterizes a part volume of the superconducting tape layer. The specific heat and density of the mass encompassed by a particular node is determined based on the ratio of the materials used in the tapes. In this case we consider the material used in the stabilizer and

$$
\left[\begin{array}{cccccc}
(1+2 F) & -F & 0 & \ldots & \ldots & 0 \\
-F & (1+2 F) & -F & 0 & \ldots & \cdot \\
0 & \cdot & \cdot & \cdot & \cdot & \cdot \\
\cdot & \cdot & \cdot & -F & (1+2 F) & -F \\
0 & \cdot & \cdot & \cdot & -F & (1+2 F)
\end{array}\right] \times\left[\begin{array}{c}
T_{1}^{t+1} \\
T_{2}^{t+1} \\
\cdot \\
\cdot \\
T_{n}^{t+1}
\end{array}\right]+\left[\begin{array}{c}
K \\
0 \\
\cdot \\
0 \\
K
\end{array}\right]
$$$$
=\left[\begin{array}{c}
T_{1}^{t} \\
T_{2}^{t} \\
\cdot \\
\cdot \\
T_{n}^{t}
\end{array}\right]+\frac{\Delta t}{C_{p} \rho} \times\left[\begin{array}{c}
Q_{1}^{t} \\
Q_{2}^{t} \\
\cdot \\
\cdot \\
Q_{n}^{t}
\end{array}\right]
$$
the substrate. This approach has previously been demonstrated in [13]. Each tape is assumed to be $4 \mathrm{~mm} \times 0.1 \mathrm{~mm}$ with a mean critical current of $100 \mathrm{~A}$ as this is a standard tape measurement. The deviation of this current from the mean value, due to inhomogenities caused by the construction of the tape and the operating conditions, is calculated based on a normal Gaussian distribution shown in Equation 6 where $\mu$ is the mean critical current (A), $\sigma$ is the standard deviation, and $I_{p}$ is the list of possible critical currents (A),

$$
I_{c}(i)=f(I p \mid \mu, \sigma)=\frac{1}{\sigma 2 \pi} e^{\frac{-\left(I_{p}-\mu\right)^{2}}{2 \sigma^{2}}}
$$

This variation in critical current levels of the superconducting material is the primary cause of the formation of hotspots, as different parts of the component are operating outside of the critical surface at different operating conditions, creating partial quench. This distribution is discretized and then sampled using a roulette wheel selection method to populate the cable with a variety of critical current levels. Figure 1 shows an example result of this selection method to populate the critical current of different sections of cable and compares the result to the normal distribution. The number of tapes required is determined by the maximum load current expected based on the power rating of the line and the voltage of the system. Additionally, it is assumed that the line incorporates a current stability margin such that the maximum load current is $60 \%$ of the critical current.

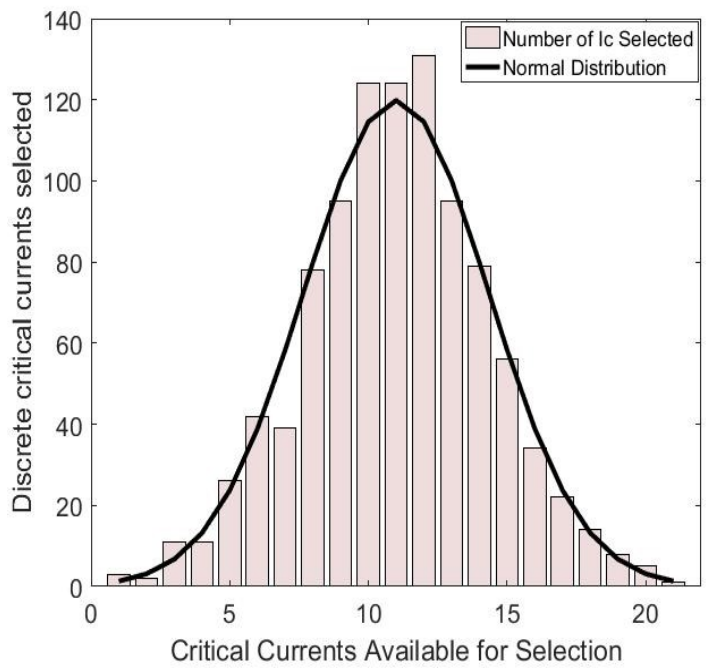

Figure 1. Discretization of critical current levels to be interleaved throughout the superconducting cable.

The resistance of the cable former is calculated using the cable dimensions and the temperature dependent resistivity of the conventional material used in the former. The former, $R_{\text {Former }}$, is considered to be to be in parallel with the HTS tapes. The HTS tapes are considered to be a mesh network where each node is represented by the superconducting material, $R_{S C}$, (YBCO) in parallel with the stabilizing layer, $R_{S T A B}$, that is composed of a conventional material, as shown in Figure 2. Contact resistance between the superconducting layer and the stabilizer is ignored. The impedance of the superconducting material at each node is calculated according to the $E-J$ characteristic described in Equation 7, where $E_{0}$ is taken as $1 \mu \mathrm{V} / \mathrm{cm}, n$ is the index of transition, $J_{c}(T, x)$ is the temperature dependent critical current density at a given node $i$, and $J(t)$ is the current density within the superconducting layer at a particular time.

$$
E(J, i)=E_{0}\left[\frac{J(t)}{J_{c}(T, i)}\right]^{n} .
$$


Current sharing between the cable former and the HTS tape, the superconducting material and the stabilizing layer at each node is calculated using an iterative current method as described in [9]. The method iteratively calculates the new current in each of the conduction layers and compares it to the previously known value, repeating this process until the error between the two is below a defined error threshold [9]. This method provides good solutions to the current sharing problem but is a relatively slow method, due to the high number of calculations which must be performed [9]. The more discretized points along the length of the cable that are selected, the longer the process takes.

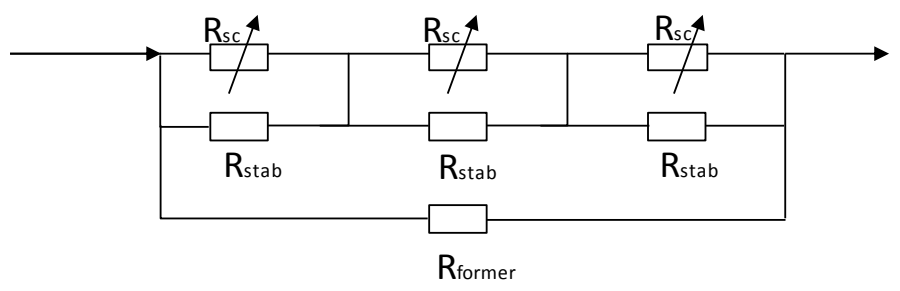

Figure 2. Example of electrical circuit discretization used in the model, in which the tapes are made of a mesh network of superconducting and conventional materials while being electrically paralleled with a former.

In order to reduce the simulation time for long lengths of cable it is assumed that cable critical currents, and associated thermal profile, repeat in a constant manner. A drawback of this assumption is that it does not consider variations in coolant temperature along a significant length of cable. These could affect the initial operating temperature of the superconducting material, directly impacting the critical current level of that section. The temperature dependence of the critical current is represented by Equation 8 where $T_{c}(i)(K)$ is the critical temperature of node $i . T_{r}$ is the reference temperature, in this case $77 \mathrm{~K}$ (the temperature of the liquid nitrogen) and $J_{c r}$ is the critical current of node $i$.

$$
J_{c}\left(T_{i}\right)=J_{c r}\left[\frac{T_{c}(i)-T(t, i)}{T_{c}(i)-T_{r}}\right]^{1.5}
$$

The temperature dependence of the transition index, $n$, is calculated according to Equation 9 [14], where $n_{o}$ is the transition index at $77 \mathrm{~K}$ and $\beta$ is the index coefficient, taken as 0.25 .

$$
n(i)=n_{o}(i)\left[\frac{T_{c}-T(i)}{T_{c}-T_{L N 2}}\right]^{\beta}
$$

\section{Cable Design Choices}

This paper considers two different designs of cable: fault current tolerant (FCT) and fault current limiting (FCL). The major difference between the two design choices is in the quenched impendence of the cable.

For the FCT cable, the former is made of highly conductive material such as copper, with a large enough cross sectional area to provide a low impedance path for the fault current to pass through during a fault scenario. The former is sized such that it contains a large volume in conjunction with the low impedance. During a fault, the amount of energy dissipated does not result in a significant rise in temperature. Hence it is possible that the cable may not experience a temperature rise great enough to cause a quench [15]. If quench is experienced, the time for the cable to recover will be reduced due to the lower temperature reached during the fault period. The large copper former of an FCT cable can reduce the possibility of a hotspot occurring as excess current above critical current will be more quickly transferred to the low impedance former [15].

While a low impedance path can offer stability during faults and small overcurrent transients, the size of the former may introduce a significant weight and volume penalty. Another disadvantage of this design is that the low impedance provided by the cable could lead to other components on the network dissipating a larger proportion of fault energy [16]. This will require other system components to be overrated to withstand the higher fault currents, incurring size and weight penalties which have a large impact on the performance (weight and efficiency) of the aircraft.

An alternative cable design is the FCL. The FCL design uses materials with much higher impedance such as stainless steel for the tape stabilizer and cable former. Hence, if fault current causes the superconducting material to go above the critical surface, it must travel through a highly resistive former. The resistive former can be sized to provide a large impedance that 
can limit the amount of fault current able to travel through the cable, reducing the amount of fault current experienced by other power system components [15]. The increased resistance may also improve the stability of the network during fault conditions.

The possibility to use the FCL cable design in a TeDP architecture will depend strongly on the available length of cable runs on the aircraft because the cable must have sufficient volume to area ratio to provide sufficient impedance while ensuring that the resulting temperature rise is not excessive. The resistive nature of the former used in the FCL design results in a greater possibility of hotspots occurring when the current levels are elevated. This is because of the reduced current sharing with the former during the initial moments of a fault and the significantly lower thermal conductivity (two orders of magnitude lower than that of copper) [17]. Hence the spread of the normal zone created by the hotspot could be exhausted, leading to a smaller volume of material absorbing the fault energy and spreading its heat to the surrounding zone much slower, reducing normal zone propagation velocity (NZPV). Finally, from a protection perspective, FCL cables are 'one-shot' devices. In this sense, FCL cables will require time to re-cool to normal operating temperatures before being placed back into service. This may take a more significant period of time to achieve than in FCT designs, owing to the larger temperature rise during the fault period and the cooling required to resume the superconducting state. Because of the limitation of the cable run length, to maintain the FCL capability, dedicated FCL devices may be required to operate with FCT cables and/or converters with FCL capability may be required (if a TeDP architecture requiring power electronic converters is selected).

\section{Simulation Results and Analysis}

\section{Hotspot Simulation Parameters}

In order to investigate the formation of hotspots, FCL and FCT cable models were developed for cables rated at $10 \mathrm{MW}$. This power rating was chosen based on published TeDP architectures [18]. For this cable, the former was sized to withstand a fault current 3.5 times larger than the full load current, and to withstand this current for approximately $1 \mathrm{~s}$ without temperature rising by more than 5 Kelvin. This cable was sized by the authors using a Monte Carlo method to ensure that the former cross sectional area (CSA) was minimized in accordance with these constraints. The stabilization layer of the superconducting tapes was assumed to be $50 \mu \mathrm{m}$ of copper. It was assumed that the total cable length is $100 \mathrm{~m}$.

For the FCL cable design, the former was assumed to be made of stainless steel. The cross sectional area was sized to provide an impedance that limits the fault current to 3.5 times full load current. This was calculated as $76.3 \mathrm{~mm}^{2}$ for a $100 \mathrm{~m}$ cable. The stabilization layer of the FCL cable design also uses stainless steel. The temperature was not allowed to exceed $150 \mathrm{~K}$ during the hotspot tests for both cables. This is considered a safe hot spot limit for many low temperature superconducting components [19], although degradation of critical current is unlikely to occur below $400 \mathrm{~K}$ in YBCO Superconductors [20]. Beyond this point, the computational efficiency of the simulations also rapidly decreased. Upon reaching this condition, the simulation would be curtailed prematurely. The superconductor considered for both the FCL and FCT cables was YBCO with a presumed critical temperature of $93 \mathrm{~K}$ and initial transition index of 21.

The hotspot simulations are carried out considering constant current levels exceeding the critical threshold of the cable by $10 \%, 20 \%, 30 \%, 40 \%$ and $50 \%$ respectively. To consider the likely possibility of improvements in the handling and manufacturing processes of superconductors reducing the amount of inhomogenities present, the measure of variance inherent in the sample was varied. However, it should be noted that due to the formation of the superconducting materials it is unlikely that inhomogeneity will ever be completely eliminated. This was achieved in the model by altering the standard deviation (SD) from the mean $I_{C}$ from 0.06 to 0.01 in steps of 0.01 . The tapes are discretized in $1 \mathrm{~mm}$ intervals with critical currents interleaved to each node. This was initially carried out for 1000 nodes to produce a model $1 \mathrm{~m}$ long. This model was then subsequently cloned 100 times under the assumption that the inhomogenities are repetitive, to represent the response of a $100 \mathrm{~m}$ long cable.

The voltage initially considered for the system was $5 \mathrm{kV}$ as this has been previously found to be an appropriate voltage for a TeDP electrical power system with a DC distribution network [6]. The operating current and cable critical current are derived from the rated power and voltage for the cable. Using the standard deviation selection, the critical currents are interleaved. The critical current profile is shown in Figure 3 for the case with a standard deviation of 0.06 . Following the worst case considered, $\mathrm{SD}$ of 0.06 with an overcurrent of $1.5 I_{C}$ that lasts 0.1 seconds, the temperature profiles observed for each of the cable designs are shown in Figures 4 and 5.

On inspection of these graphs it can be seen that following the application of the overcurrent, the superconducting layer of the FCT has a very smooth temperature profile that peaks close to $79.75 \mathrm{~K}$, far below the critical temperature. This smooth temperature profile can be attributed to the high thermal conductivity of the copper stabilizer present in the superconducting tapes. In addition, as a result of the low impedance of the copper former, there is less current flowing through the superconducting tapes, with this current being diverted into the former. Due to the thermal inertia of the copper former, the contribution that its temperature rise has to the superconducting tapes is considered negligible over the relatively short timescales considered. This was in stark contrast to the FCL cable design. Due to the low thermal conductivity of the stabilizer, the hotspots occur in clear isolation and reach relatively high temperatures. The large thermal gradient created by these locations could potentially cause wear and tear through crack growth. 


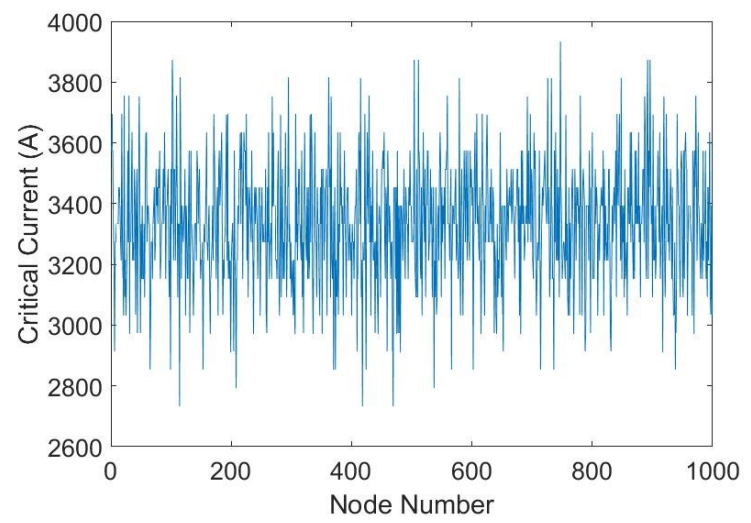

Figure 3. Critical current distribution for $5 \mathrm{kV}$ cable used in simulations.

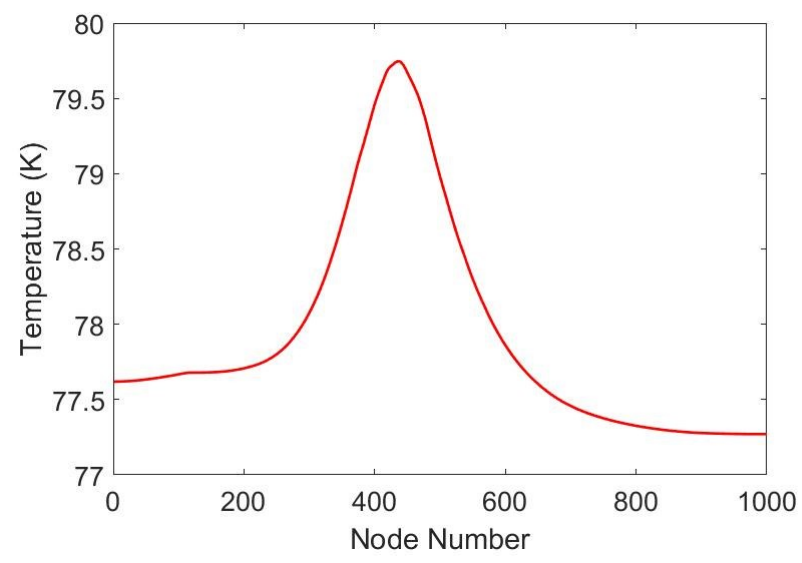

Figure 4. Temperature distribution of superconducting layer in FCT cable following overcurrent simulation.

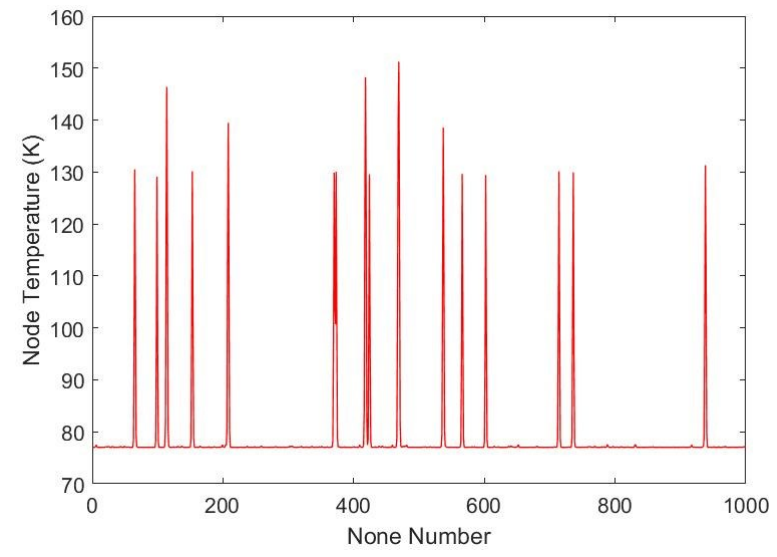

Figure 5. Temperature distribution of the superconducting layer in FCL cable following overcurrent simulation.

\section{FCT Cable Response}

The impact of varying the magnitude of the standard deviation and the overcurrent on the FCT cable temperature profile is shown in Figures 6 and 7 respectively. It can be seen from Figure 6 that reducing the standard deviation, the variability of the critical current, while keeping the overcurrent magnitude constant, has a positive effect on reducing the maximum hotspot temperature as well as smoothing the components temperature profile. Figure 7 shows that by reducing the magnitude of the overcurrent, the peak temperature was reduced, although the shape of the temperature profile was still non-uniform. The largest temperature rise is generally between nodes 300 to 600 , while nodes 0 to 300 experience a moderate increase and nodes 600 to 1000 undergoing only minor temperature rise. This is because there is less current flowing in the resistive part of the superconducting tapes in these areas, leading to reduced Ohmic losses.

Although the temperature rise appears insignificant for the FCT design, it has a significant impact on the critical current profile of the cable due to the temperature dependency of the critical current. The critical current profile following the overcurrent 
for the worst case scenario is shown in Figure 8. At it is most severe point, the critical current is reduced to $75.4 \%$ of its initial value. Given that the assumed stability margin of the cable is $60 \%$ of it is maximum capacity, the full load current is now much closer to the critical current limit. This will lead to greater heat losses, increasing the cooling system requirements. In addition, the cable will now be more susceptible to future faults until it returns to its normal operating temperature due to it operating so close to the critical current. This could impact on protection operating speed requirements. Indeed, these may need to be preemptively set to account for the potential variation in, or dynamically adjusted in response to, changing thermal and electrical conditions. To achieve this new detection methods will need to be developed.

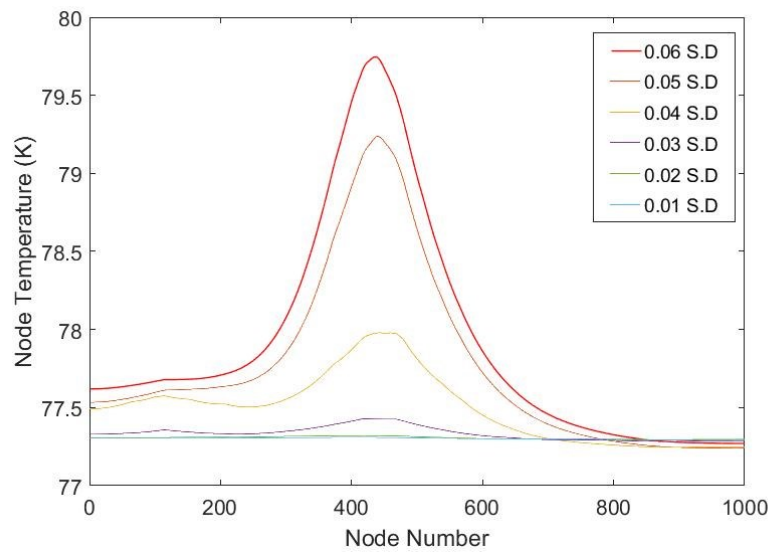

Figure 6. Temperature distribution in FCT cable at a variety of cable inhomogeneity levels.

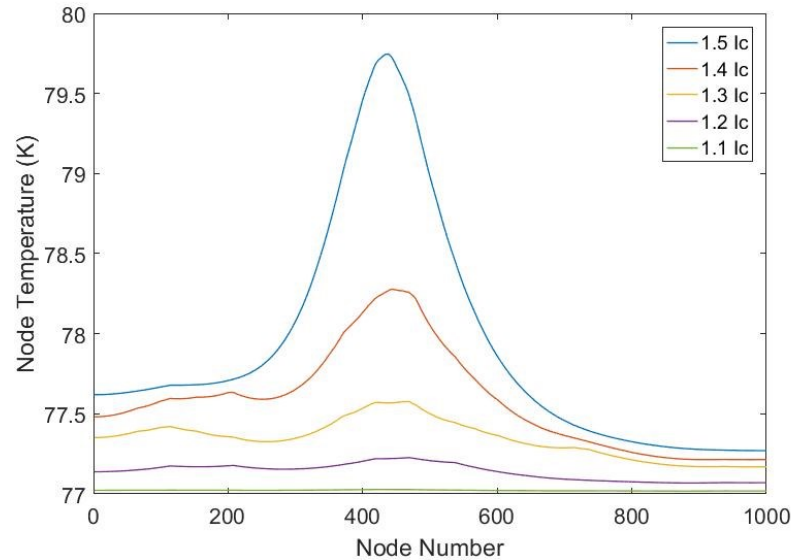

Figure 7. Temperature distribution in FCT cable at a variety of overcurrent levels.

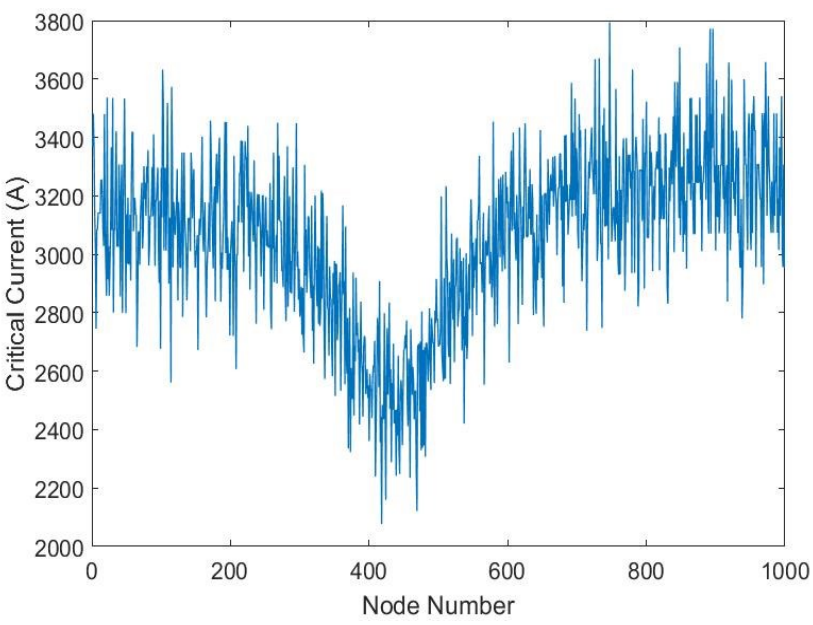

Figure 8. Cable critical current distribution following overcurrent simulation. 


\section{FCL Cable Response}

For the FCL cable design a similar effect can be seen. Figures 9 and 10 show the temperature profile of the FCL cable with a standard deviation of 0.01 and 0.03 respectively. Note that overlaid plots of temperature are not utilised here (as per the results for the FCT cable) as this would result in a loss of visual clarity. Separate traces for individual cases are instead presented. It can be seen that hotspots still occur in the cable with the lower modelled SD value, but that there are fewer of them in comparison to the cable with the higher SD value. This could result in hotspots becoming more difficult to detect because there is a smaller zone dissipating heat and thus producing a less noticeable voltage drop. Given that temperature will not be a usable quantity for fault detection over timescales of less than 1 second, new detection methods will likely be required to ensure component failure modes such as this can be captured.

Variations in critical current will be extremely challenging to eliminate as they arise due to manufacturing defects, poor handling, and operating conditions. There are however, a number of methods that can be applied to reduce the impact of hotspots such as using a stabilizer with a greater conductance. Another method suggested by the authors of [21] involves the use of a parallel shunt tape that can connect tapes on the superconducting layer together, allowing current to be redistributed amongst the superconducting tapes during transmission across the cable. This method can be shown to aid in equalizing the quench profile experienced by the individual tapes [21].

The temperature profiles of the FCL cable with a standard deviation in critical current variation of 0.06 and following overcurrent transients of $1.1 I_{C}$ and $1.3 I_{C}$ are shown in Figures 11 and 12 respectively. It can be seen from Figure 11 that the lower current test has caused the cable temperature to rise only slightly above the coolant temperature $(77 \mathrm{~K})$, indicating some stability of this cable configuration to lower current values for a limited time. For the $1.3 I_{C}$ test illustrated in Figure 12, the peak temperature of the cable reaches $150 \mathrm{~K}$, although the temperatures at other locations are comparatively lower than those shown in Figure 5, for the worst case scenario (with $1.5 I_{C}$ and an SD of 0.06). This is expected due to lower current sharing between the stabilizing layers within the tapes.

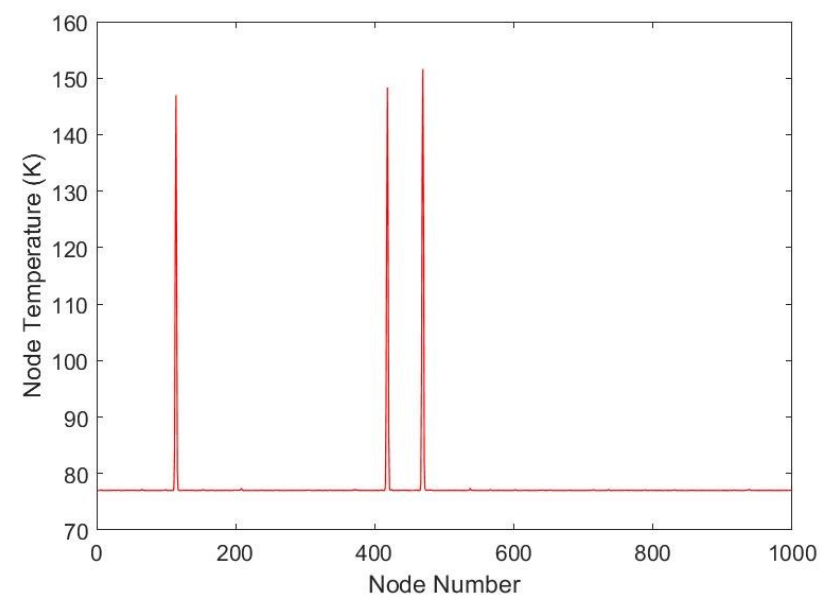

Figure 9. Temperature profile following $1.5 I_{C}$ overcurrent in FCL cable with 0.01 standard deviation of $I_{C}$

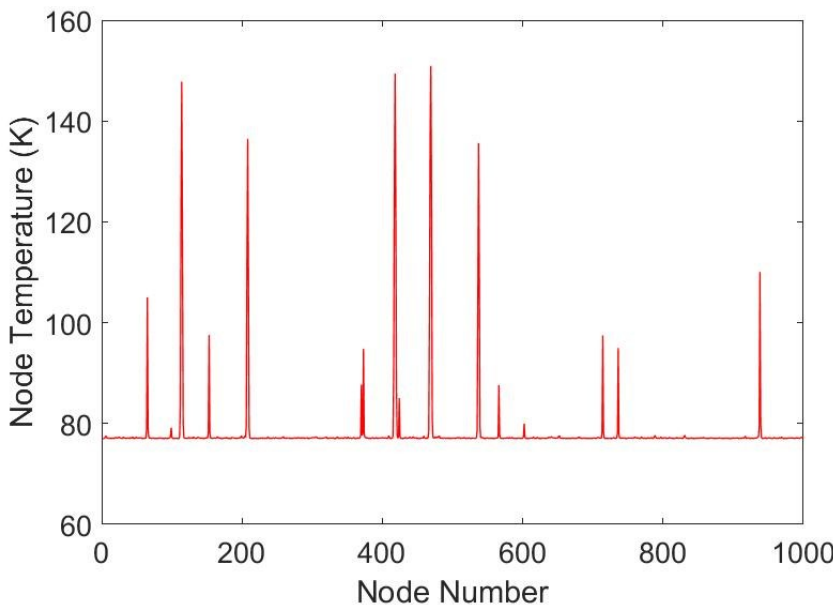




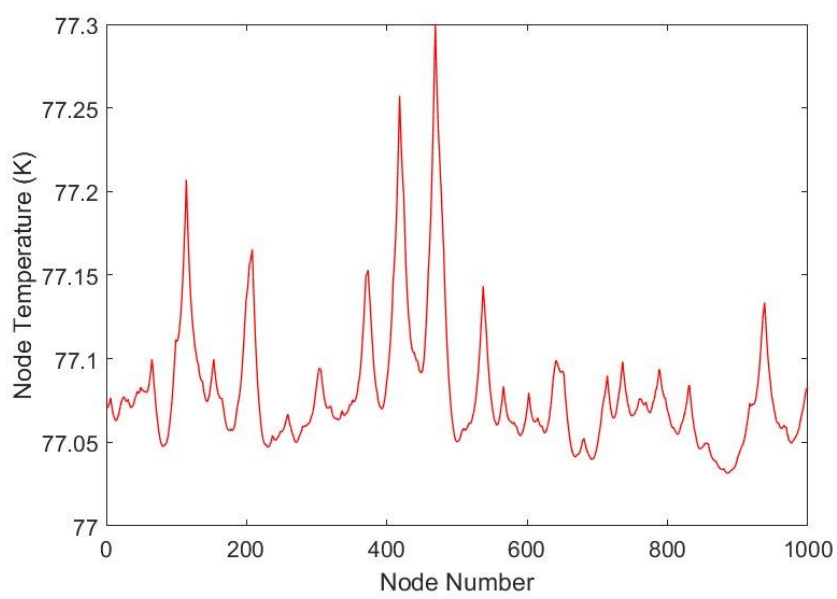

Figure 11. Temperature profile following $1.1 I_{C}$ overcurrent in FCL cable with 0.06 standard deviation of $I_{C}$

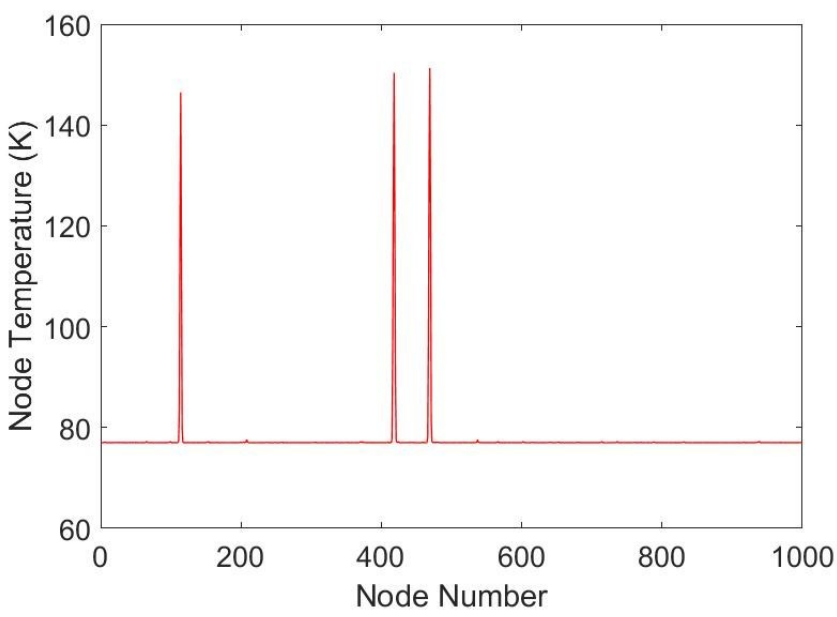

Figure 12. Temperature profile following $1.3 I_{C}$ overcurrent in FCL cable with 0.06 standard deviation of $I_{C}$

\section{Impact of Voltage Level on Cable Performance}

The electrical power system architecture for TeDP is still an area of ongoing research. Therefore, it is important to consider that the system may operate at other voltages to take advantage of certain effects. For instance, lower voltage systems could potentially better exploit the power density of superconducting cables while higher voltage systems could serve to reduce switching losses within power converters.

To investigate this, a sweep of simulations was carried out to consider the impact of varying the system operating voltage (with a constant power rating of $10 \mathrm{MW}$ ) from $1 \mathrm{kV}$ to $10 \mathrm{kV}$ on the maximum cable temperature after a fault event. The discrete voltages used in these simulations were $1 \mathrm{kV}, 2.5 \mathrm{kV}, 5 \mathrm{kV}, 7.5 \mathrm{kV}$ and $10 \mathrm{kV}$. In addition, the fault current peak (as a multiple of the critical current $I_{C}$ ) and standard deviation of critical current along the cable were varied in the same manner and over the same range as for the previous case studies presented. For each voltage level, the cable formers are resized in accordance with the requirements set out in the previous section. In each case the number of superconducting tapes used is selected to provide the same stability margin for full load current calculated assuming a $10 \mathrm{MW}$ cable operating at the selected voltage level. It is assumed that for higher current requirements a single cable is used rather than parallel arrangements, which may well be the case in the final design.

The results of these simulations are shown in Figures 13 and 14 for the FCT and FCL designs respectively, which display the variation in peak cable temperature as a function of the operating voltage, fault current peak and critical current SD. 
It can be seen from Figure 13 that the peak temperature of the FCT design remains below its critical temperature for the full range of voltages considered. Larger overcurrents and greater critical current variations can still be seen to cause a higher peak

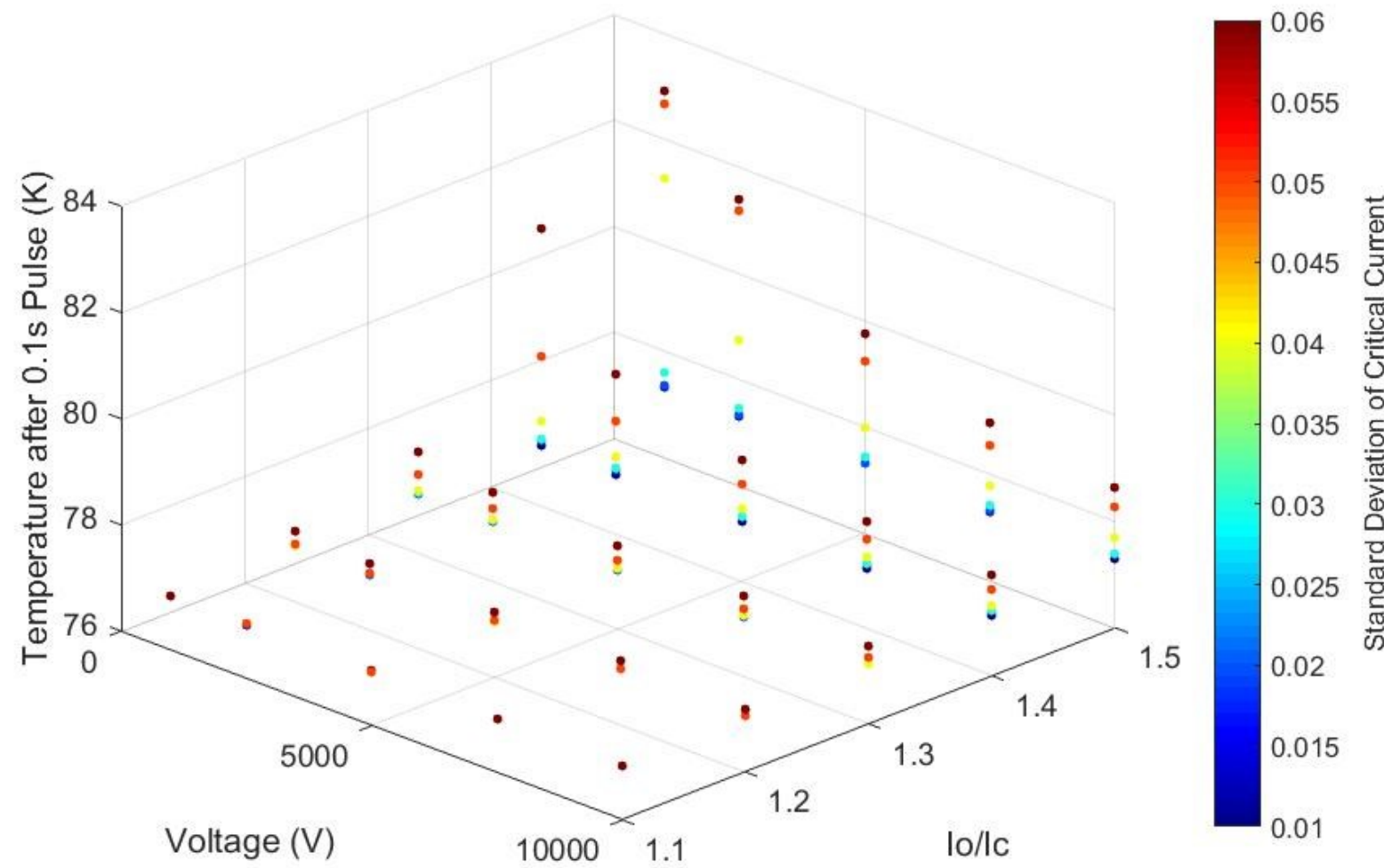

temperature within the component though. In the worst case scenario considered, the highest temperature reached is approximately $83 \mathrm{~K}$. This would have the effect of reducing the critical current significantly in the area concerned, which may necessitate removing the component from operation until normal operating temperatures are restored. Alternatively, the maximum operating current of the component could be reduced through adaptive converter control. This localized heating behavior may also necessitate a larger stability margin in setting the $I_{C}$ of the cable to account for transient events, leading to a greater amount of superconducting material used in the component. It can be seen from Figure 13 that localized heating effects

Figure 13. Peak temperature in superconducting layer following variable overcurrent pulse in FCT cable.

are exacerbated at lower operating voltages. This is because the assumed operating current is much larger and therefore losses due to heat increase.

Figure 14 shows the variation in peak cable temperature as a function of the operating voltage, fault current peak and critical current SD for the FCL design. At overcurrent levels above 1.1 times the critical current, the superconducting layer is seen to quench at higher values of inhomogeneity. Indeed, most simulations above this current cause the hotspot temperature to reach the limit of the simulation, $150 \mathrm{~K}$.

Figure 15 shows the time taken for the temperature to reach the thermal limit as a function of the operating voltage, fault current peak and critical current SD. Note that an illustrated time of 0.1 seconds indicates the limit is not reached in the simulation. It can be seen from this graph that the most important factor in determining how long it takes for the superconductor to reach the thermal limit is the overcurrent level. Higher operating currents associated with lower voltages will lead to quicker quench inception, which occurs in nearly all cases of fault current magnitudes greater than 1.1 times the critical current. 


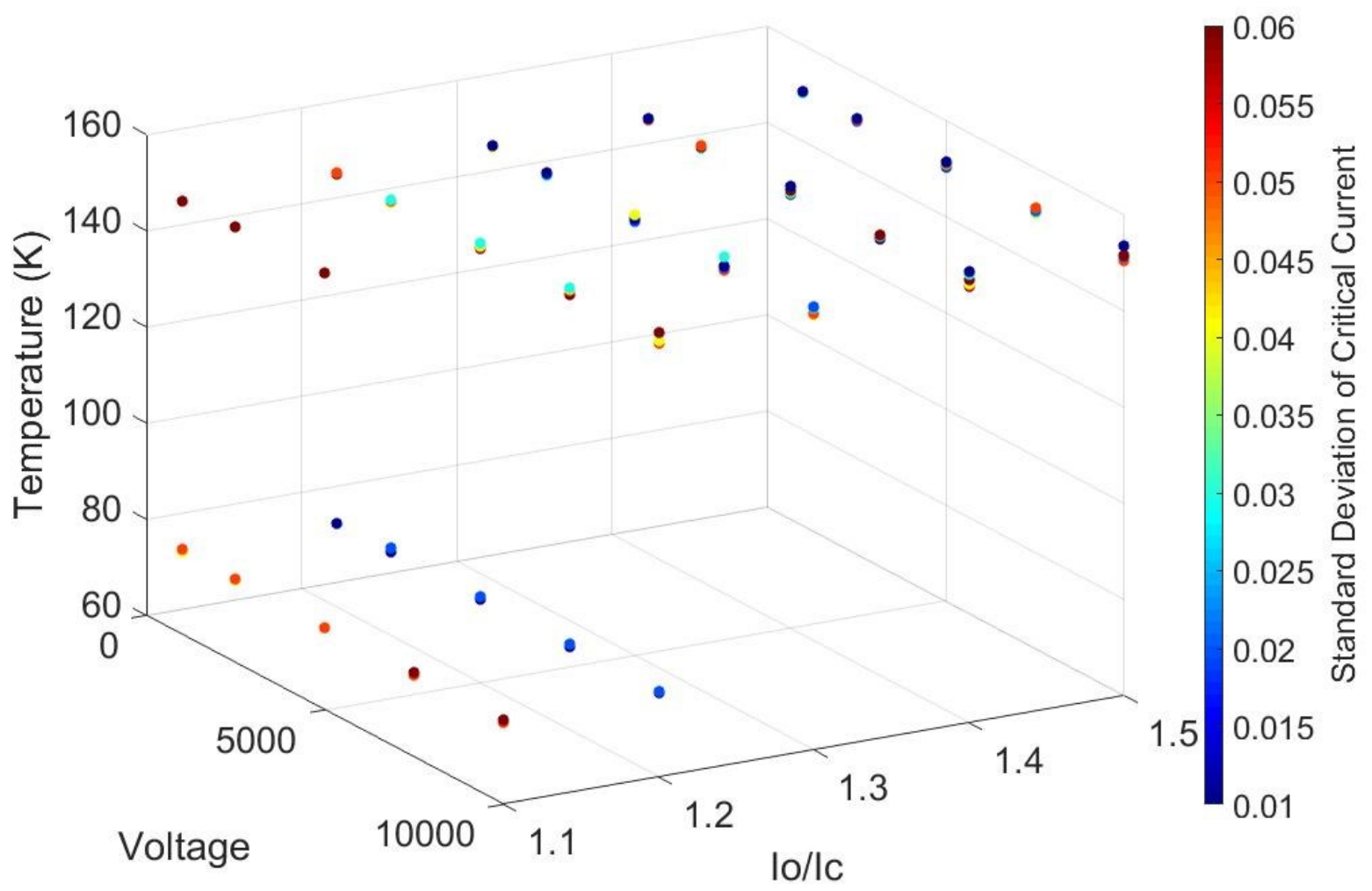

Figure 14. Peak temperature in superconducting layer following variable overcurrent pulse in FCL cable.

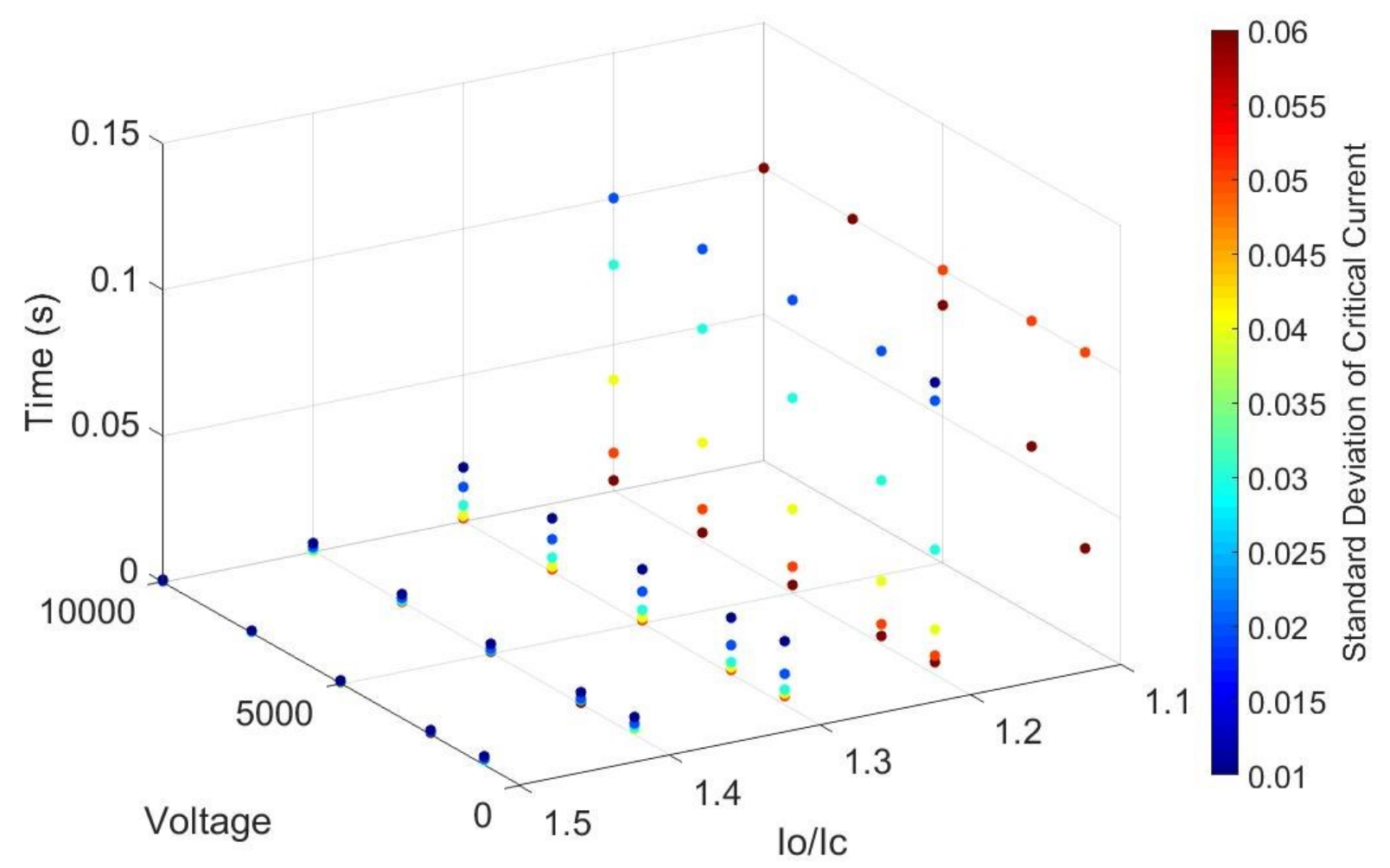

Figure 15. Time taken for the overcurrent pulse to cause the peak temperature in the FCL cable to rise above the $150 \mathrm{~K}$ limit for the simulation. 
In order to overcome this disadvantage of the FCL, it may be necessary to use a larger stabilizer with a higher thermal conductivity. However, this is complicated by the small cable lengths in an aircraft application, as it would be difficult to provide the necessary resistance required to provide damping to the fault current within the associated cable length. Alternatively, a larger stability margin could be used to avoid the hotspot formation. However, this may reduce the response time of fault current limiting function of the cable when it is required, limiting the effectiveness of the cable as a protection device.

\section{Fault Current Limiting Effectiveness of an FCL Cable}

To test the fault current limitation capability of an FCL superconducting cable on board a TeDP aircraft, this section considers a rail to rail short circuit condition. This simulation assumes an ideal DC voltage source providing the fault current and therefore ignores the wider systems affects that might influence the response such as the discharging of capacitors and the resulting voltage collapse. To this end the instantaneous fault current is calculated according to Equation 10 where $I_{F}$ is the new instantaneous fault current, $L$ is the inductance of the line (assumed to be $100 \mu \mathrm{H}$ ) $R_{F C L}$ is the resistance of the superconductor and $V_{0}$ is the operating voltage.

$$
I_{f}(t+1)=\int_{o}^{t} \frac{1}{L}\left(V_{0}-i(t) R_{F C L}(t)\right) d t
$$

The range of values used for the initial voltages in these simulations is the same as in the hotspot simulations. The simulation run time is set to $500 \mathrm{~ms}$ although the simulation ends if the peak temperature of the tapes or the former exceeds $300 \mathrm{~K}$ (room temperature). As before, this temperature is selected as it presents a convenient benchmark beyond which computational efficiency of the simulations decreases rapidly. The inhomogeneity level is kept to a standard deviation of 0.01 for all simulations. It is assumed that the fault happens while a full load current is being drawn through the cables.

Figure 16 shows the temperature profiles of the superconducting tapes in the FCL cables for the range of operating voltages considered. It can be seen that the temperature remains below the $I_{C}$ degradation point throughout the superconducting tapes, even after the former reaches $300 \mathrm{~K}$, although protection would have to act quickly to prevent further heating and transfer of thermal energy to the superconducting tapes. The temperature profile of the tapes is smoother compared to previous tests with small overcurrent levels, although there are clear areas where very large thermal gradients exist. This is because the fault current is large enough to quench most of the superconducting layer in unison before the majority of the current begins to travel in the former material.

The cable former, which transports most of the current following the quench, dissipates much more of the heat in a fault scenario, causing it to reach a relatively high temperature. Further studies would be required to see if such a sudden and large temperature rise would exert too much thermal strain on the materials involved. It should also be noted that this simulation assumes a $100 \mathrm{~m}$ cable length which may not commonly occur on an aircraft. Reducing the length would require the former cross sectional area to also be reduced to provide the same damping, greatly limiting the volumetric heat capacity available. This in turn means that a larger temperature rise would be observed in the cable former in the same time period.

Figure 17 shows the peak temperature reached by the former of the cables as a function of the operating voltage. The time taken for this to occur is also shown. From this plot, it can be seen that for all operating voltages above $2.5 \mathrm{kV}$, the peak temperature in the former reaches the maximum temperature allowed by the simulation. This is in contrast to the previous simulations where small overcurrents caused lower voltage systems to quench faster. The reason this occurs is two-fold. Firstly, the larger overcurrent caused by a high-voltage fault in combination with the lower $I_{c}$ of the superconducting layer helps with uniformity of quench. This can be seen in the temperature profiles of the superconducting layers on Figure 16. Secondly, the higher operating voltages require progressively higher resistance formers to be utilised to provide sufficient fault current limiting (i.e. maintaining a constant $\mathrm{V} / \mathrm{R}$ ratio). This leads to a smaller cross-sectional area of the former, effectively reducing the volume available to dissipate the heat energy of the fault current, causing swift temperature rise in the former, Figure 17.

Figure 18 shows the transient current profile through an FCL cable following the occurrence of the fault, with a network operating voltage of $1 \mathrm{kV}$. In order to ensure that the cables are not damaged, protection systems will be need to be able to react within these timeframes to interrupt the fault current. 


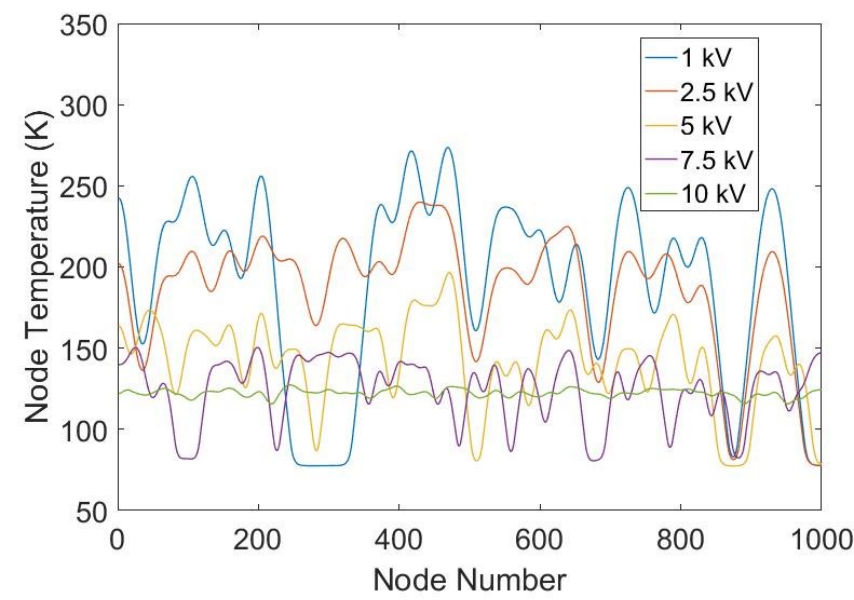

Figure 16. The Temperature profile of the superconducting tapes following fault current test in the FCL cable.

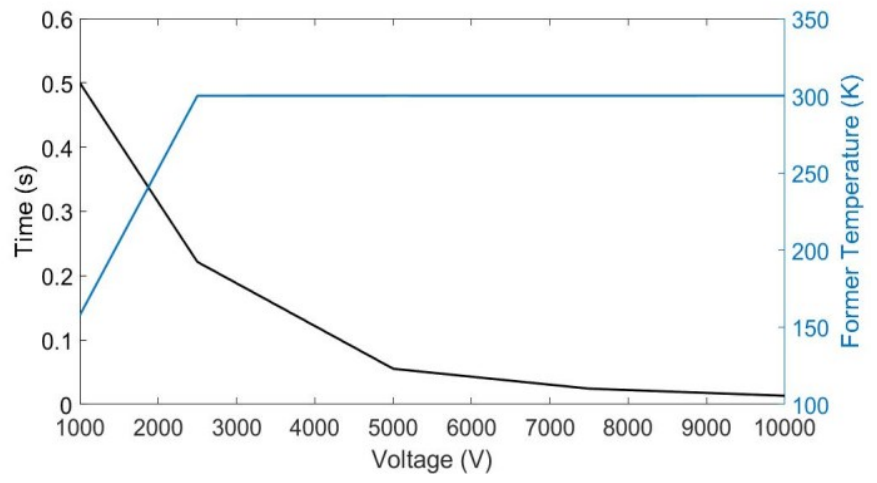

Figure 17. Time taken for fault current to cause temperature rise to reach $300 \mathrm{~K}$ limit (black). Temperature of the former following simulation (Blue).

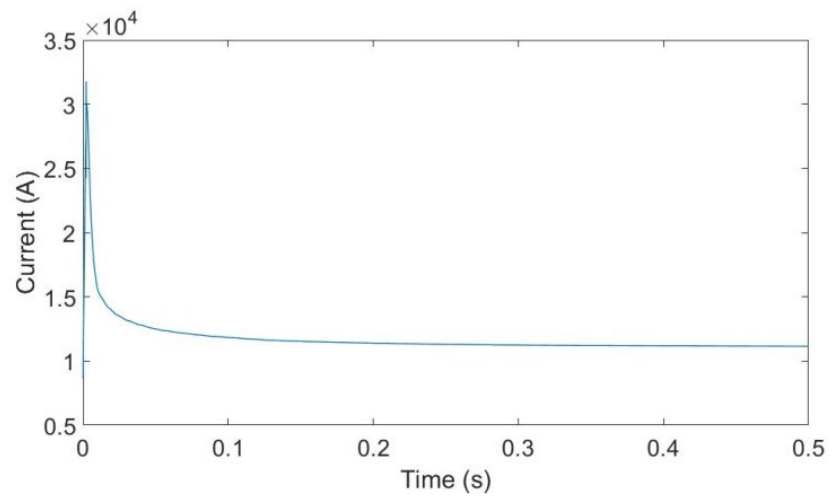

Figure 18. Fault Current profile in FCL cable for fault on system operating at $1 \mathrm{kV}$.

\section{Conclusions}

In conclusion this paper demonstrates the potential for superconducting cables to undergo partial quench, potentially requiring them to be removed from service. The severity of this can depend on whether an FCL or FCT cable design is used. In order to protect against this failure mode of the cables it may be necessary to employ new dedicated hotspot protection systems. Improvements in material homogeneity could potentially serve to reduce the extent of the damage resulting from this failure mode by allowing a more uniform quench which promotes better current sharing with the cable former. This is far more relevant for FCL designs as the temperature profiles has been shown in this paper to have a significantly larger variance than in FCT designs.

The large temperature rise shown to occur in the FCL design for all scenarios considered clearly shows the requirement for redundancy or reconfigurability in the electrical power distribution system if continued supply of electrical power is to be maintained to key loads (e.g. propulsion motors) following a fault scenario. This could add unacceptable weight penalties to the type of design that might overshadow some of the potential benefits otherwise afforded through inherent fault current limiting. 
Fault current limiting through other means such as current limiting converters could replace the requirement for the FCL cables. These devices cannot prevent the fault effects from propagating throughout a DC network, which without the inherent damping of FCL cables, bus voltage stability could still be lost, which could lead to loss of thrust.

Even if the superconducting state is not lost during the overcurrent event, the effective critical current level throughout the length of a superconducting cable can be reduced by a significant amount. In order to make components better capable for fault ride through and thereby afford the system a greater amount of stability, more superconducting material may be required to increase the critical current above the threshold requirements of faults in other parts of the system. However, this is traded against an increase in the amount of superconducting materials required. For future TeDP aircraft these protection requirements will have to be taken into consideration in order to ensure that the system remains stable at all points of the flight cycle, as well as during large transient fault conditions.

\section{References}

1. Airbus, "Flying By Numbers", Airbus, [online] Available: http://www.airbus.com/company/market/forecast/, 2015, accessed on: 12th of May 2016.

2. J. L. Felder, H. D. Kim, and G. V. Brown, "Turboelectric Distributed Propulsion Engine Cycle Analysis for HybridWing-Body Aircraft”, 47th AIAA Aerosp. Sci. Meet. Incl. New Horizons Forum Aerosp. Expo., 2009.

3. European Commission," Flightpath 2050 Europe's Vision for Aviation Report of the High Level Group on Aviation Research", European Union. Available at: http://ec.europa.eu/transport/sites/transport/files/modes/air/doc/flightpath2050.pdf Date Accesed: 03/05/2017

4. J. L. Felder, H. D. Kim, and G. V. Brown, "Distributed Turboelectric Propulsion for Hybrid Wing Body Aircraft," 9th International Powered Lift Conference, London, United Kingdom, July 2008

5. C. Friedrich, P.A. Robertson, "Hybrid-electric propulsion for automotive and aviation applications", CEAS Aeronautical Journal, Vol 6.2, Springer, Dec. 2014

6. M. J. Armstrong et al., "Architecture, voltage, and components for a turboelectric distributed propulsion electric grid", NASA/CR - 2015- 218440, [online] Available: http://ntrs.nasa.gov/archive/nasa/casi.ntrs.nasa. gov/20150014237.pdf, 2015, accessed on: 12th May 2016.

7. Kalsi, Swarn, Singh, “Applications of High Temperature Superconductors to Electric Power Equipment” Chap 4, John Wiley \& Sons Ltd, 2011

8. I. Chew, A. Lapthorn, P. Bodger and W. Enright, "Superconducting transformer failure: Testing and investigation", Australian Universities Power Engineering Conference, pp. 1-5, 2009

9. W. T. B. de Sousa, “Transient Simulations of Superconducting Fault Current Limiters”, Ph.D. dissertation, Dept. Elect. Eng, Federal University of Rio de Janeiro, Brazil, 2015.

10. D. Colangelo and B. Dutoit, "Impact of the normal zone propagation velocity of high-temperature superconducting coated conductors on resistive fault current limiters", IEEE Trans. Appl. Supercond., vol. 25, no. 2, pp. 1-7, 2015.

11. N. Nanato, Y. Tsumiyama, S.B. Kim, S. Murase, K.-C. Seong, H.-J. Kim, Development of quench protection system for HTS coils by active power method, Physica C: Superconductivity and its Applications, Volumes 463-465, 1 October 2007, Pages 1281-1284, ISSN 0921-4534, https://doi.org/10.1016/j.physc.2007.02.047.

12. J. H. Kim, C. H. Kim, V. Pothavajhala and S. V. Pamidi, "Current Sharing and Redistribution in Superconducting DC Cable," in IEEE Transactions on Applied Superconductivity, vol. 23, no. 3, pp. 4801304-4801304, June 2013.

13. G A Levin and K A Novak and P N Barnes, "The effects of superconductor-stabilizer interfacial resistance on the quench of a current-carrying coated conductor", Superconductor Science and Technology, Vol. 23 Pages 14 -21, IOP Publishing, Dec 2009.

14. D. Colangelo and B. Dutoit, "Inhomogeneity effects in HTS coated conductors used as resistive FCLs in medium voltage grids", Superconductor Science and Technology, Vol. 25 num. 9, IOP Publishing, June 2012

15. C. E. Bruzek, A. Allais, K. Allweins, D. Dickson, N. Lallouet, and E. Marzahn, "Using superconducting DC cables to improve the efficiency of electricity transmission and distribution (T\&D) networks", Superconductors in the Power Grid, Elsevier Ltd, 2015.

16. S. Nolan et al. "Understanding the impact of failure modes of cables for the design of turbo-electric distributed propulsion electrical power systems", ESARS ITEC 2016, Toulouse, France, October 2016. 
17. J.E. Jensen, W.A. Tuttle, R.B. Stewart, and H.Brechna et al. "BROOKHAVEN NATIONAL LABORATORY SELECTED CRYOGENIC DATA NOTEBOOK" United Stated Department of Energy, August 1980. Available at: https:/www.bnl.gov/magnets/staff/gupta/cryogenic-data-handbook/subject.htm

18. C. E. Jones, P. J. Norman, S. J. Galloway, M. J. Armstrong and A. M. Bollman, "Comparison of Candidate Architectures for Future Distributed Propulsion Aircraft," in IEEE Transactions on Applied Superconductivity, vol. 26, no. 6, pp. 1-9, Sept. 2016. doi: 10.1109/TASC.2016.2530696

19. Herman ten kate, "(Multi) Normal Zone Propagation Velocity in high current density high field magnets", CERN presentation, Jan 2013. Available at: https://indico.cern.ch/event/199910/contributions/381641/attachments/297506/415833/TenKate WAMSDO 2013 Propagation15Jan13.pdf

20. M. J. Kim et al., "Determination of Maximum Permissible Temperature Rise Considering Repetitive Over-Current Characteristics of YBCO Coated Conductors," in IEEE Transactions on Applied Superconductivity, vol. 18, no. 2, pp. 660-663, June 2008. doi: 10.1109/TASC.2008.921397

21. S. Venuturumilli et al., "Forceful Uniform Current Distribution Among All the Tapes of a Coaxial Cable to Enhance the Operational Current," in IEEE Transactions on Applied Superconductivity, vol. 27, no. 4, pp. 1-4, June 2017. doi: 10.1109/TASC.2016.2642138

\section{Contact Information}

Steven Nolan. $\underline{\text { Steven.nolan@strath.ac.uk }}$

Technology \& Innovation Centre, 99 George St, Glasgow G1 1RD

\section{Acknowledgments}

This work was undertaken as part of the Rolls-Royce UTC program.

\section{Definitions/Abbreviations}

TeDP

HTS

BLI

FCL

FCT

NZPV

CSA
Turbo-electric Distributed

Propulsion

High-Temperature

Superconducting

Boundary Layer Ingestion

Fault Current Limiting

Fault Current Tolerant

Normal Zone Propagation

Velocity

Cross Sectional Area 